\title{
The Effect of Neurofeedback Training on Sustain Attention and Working Memory in Male Elementary School Students with Attention-Deficit/ Hyperactivity Disorder
}

Zahra Dashtbozorgi ${ }^{1}$, Mehri Dadashpour Ahangar², Sahar Aminalsharieh ${ }^{3}$, Jamal Ashoori $^{4}$, Marjan Alizadeh ${ }^{*}$

${ }^{1}$ Department of Psychology, Ahvaz Branch, Islamic Azad University, Ahvaz, Iran

${ }^{2}$ Department of Psychology, Roodehen Branch, Islamic Azad University, Roodehen, Iran

${ }^{3}$ Department of Psychology, Khorasan Razavi Sciences and Research Branch, Islamic Azad University, Neyshaboor, Iran

${ }^{4}$ Department of Psychology, Isfahan (Khorasgan) Branch, Islamic Azad University, Isfahan, Iran

Article Info:

\section{A BSTRACT}

Introduction: Several difficulties of students with attention deficit/ hyperactivity disorder (ADHD) are related to executive dysfunctions, which may improve by neurofeedback training. This study was aimed to investigate the effect of neurofeedback training on sustain attention and working memory in male elementary school students with ADHD. Materials and Methods: This study was a quasi-experimental investigation with a pretest-posttest design. The statistical population were included male elementary school students with ADHA that referred to counseling centers of Varamin city in 2015. Thirty students were selected using sampling method and randomly divided into two groups. The experimental group educated twelve 60-minute-sessions of neurofeedback training. To assess sustain attention and working memory, CPT and N-back computerized tests were performed, respectively. Results: There was a significant difference between the averages of sustains attention and working memory of experimental and control groups in the posttest stage. The neurofeedback training significantly increased sustain attention and working memory in male elementary school students with ADHD. Conclusion: The findings suggest use of neurofeedback training to improve cognitive dysfunction in children with ADHD.

*Corresponding Author: Marjan Alizadeh

E-mail: alizadeh1531@yahoo.com 


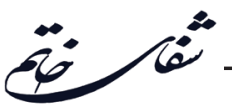

تأثير آموزش نوروفيدبك بر توجه يايدار و حافظةٌ كارى در دانش آموزان يسر ابتدايى مبتلا به اختلال نارسايى توجه /بيش

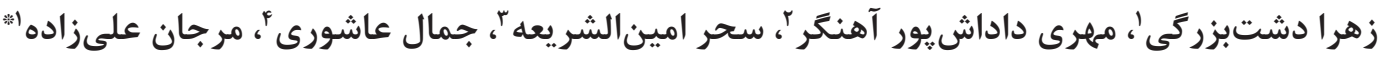

\author{
'كروه روانشناسى، واحد اهواز، دانشكاه آزاد اسلامى، اهواز، ايران

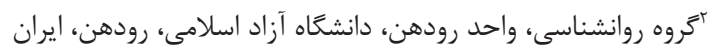

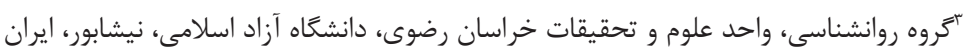

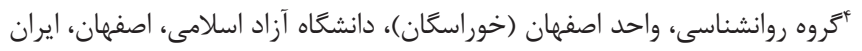

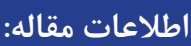

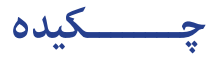

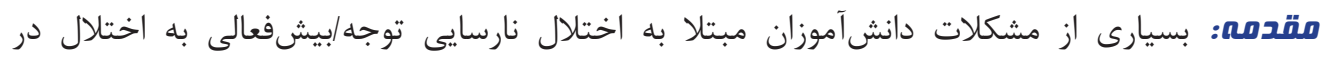

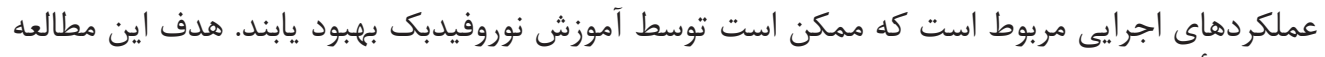
بررسى تأثير آموزش نورايى نوروفيدبك بر توجه

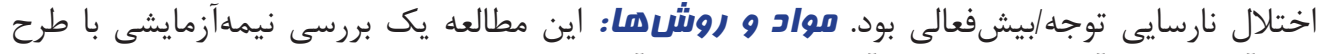

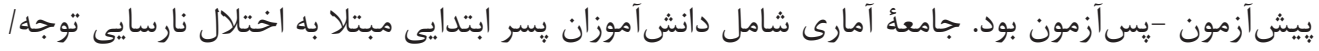

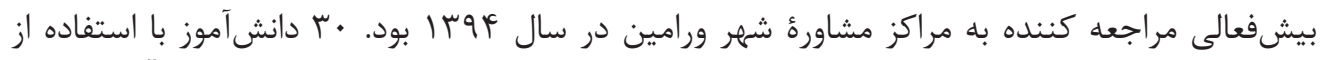

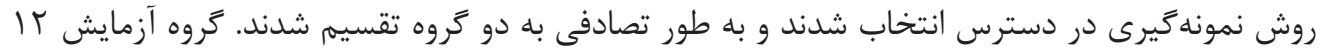

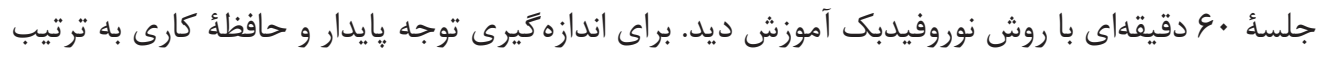

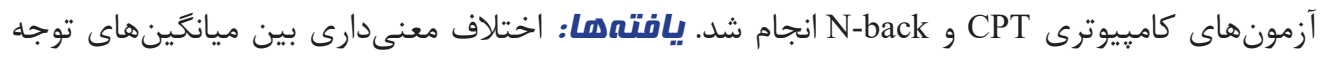

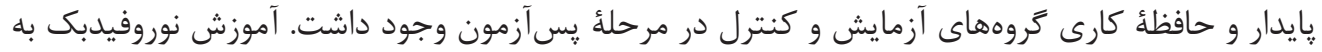

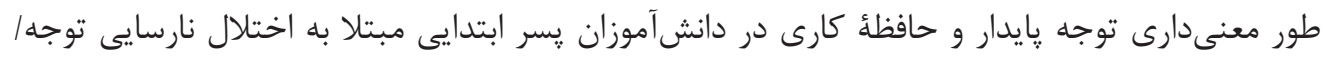

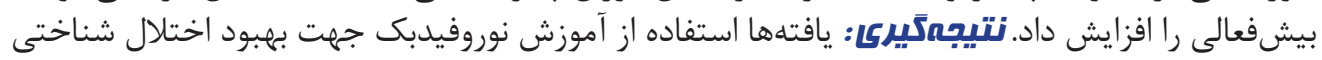

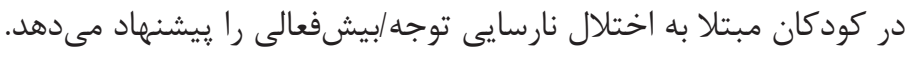

كليد وازهها:

1. نوروفيدبك

r. r. توجه

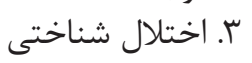

"نويسنده مسئول: مرجان علىزاده

آدرس الكترونيكى: alizadeh1531@yahoo.com 


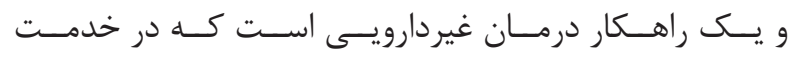

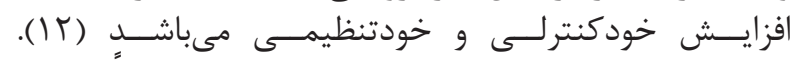

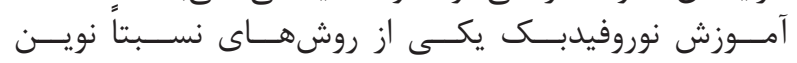

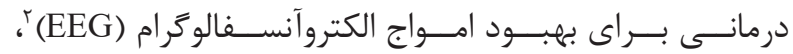

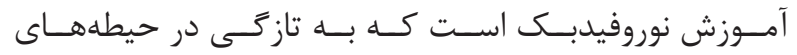

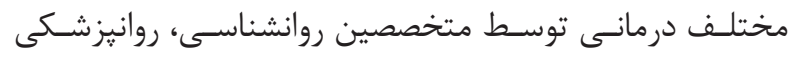

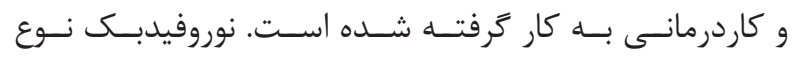

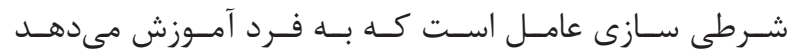

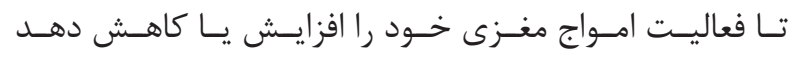

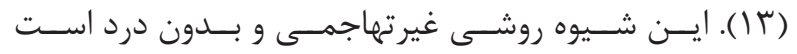

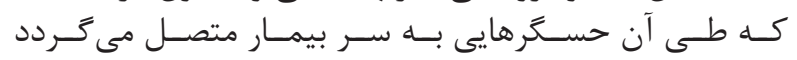

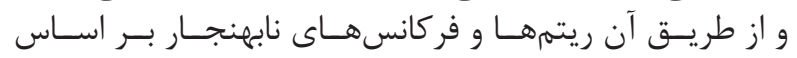

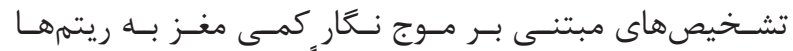

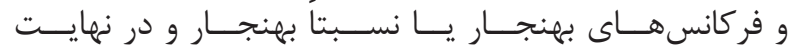

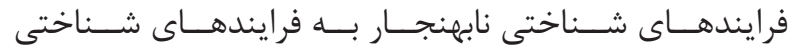

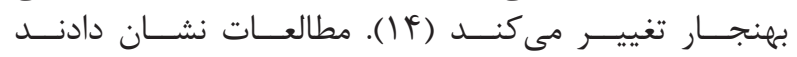

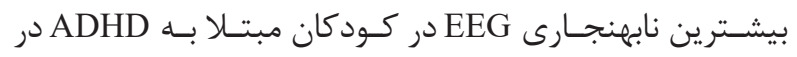

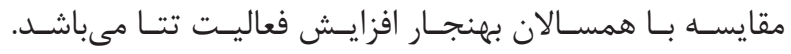

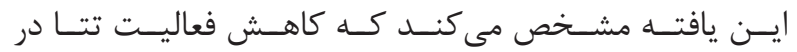

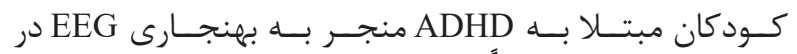

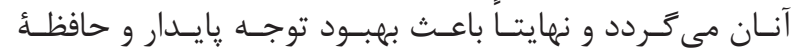

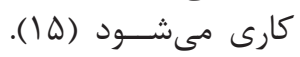

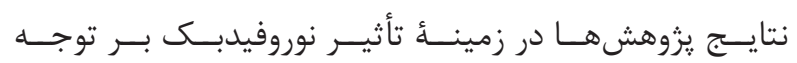

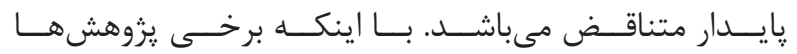

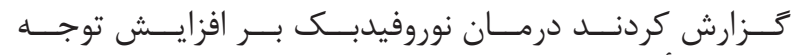

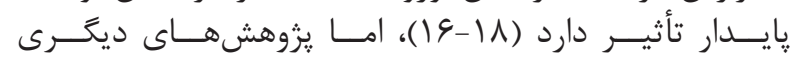

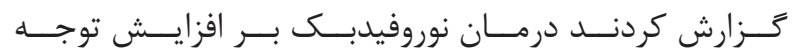

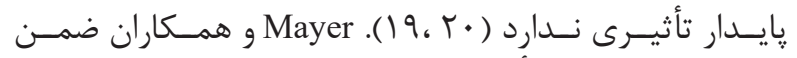

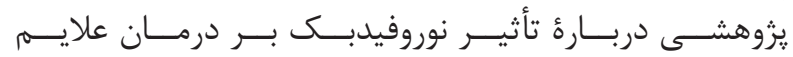
ADHD

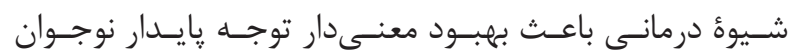

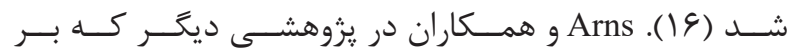

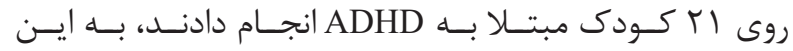

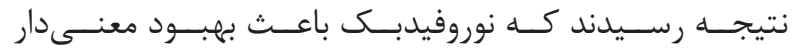

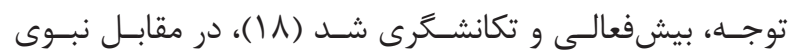

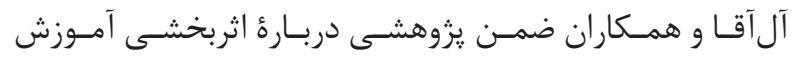

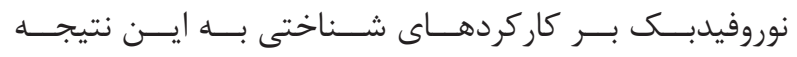

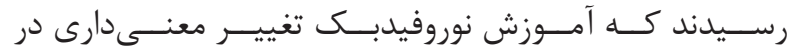

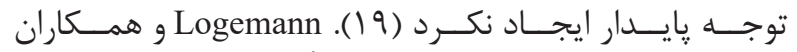

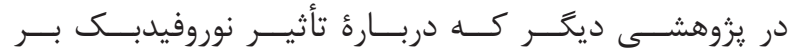

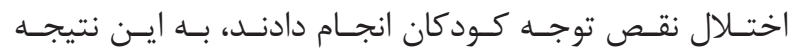

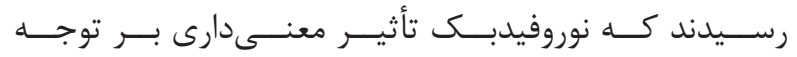

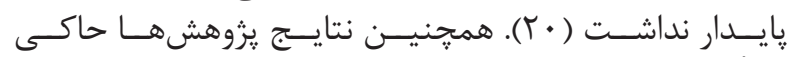

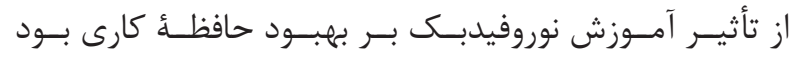

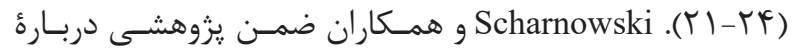

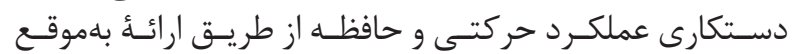

مقدمه

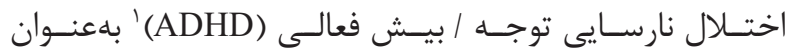

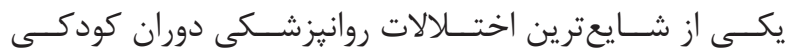

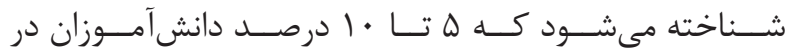

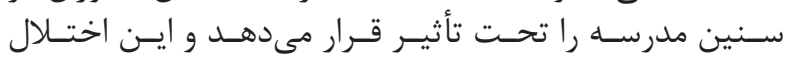

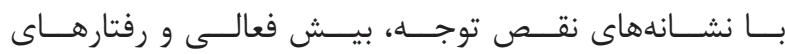

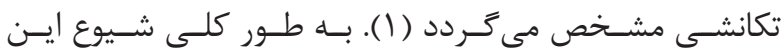

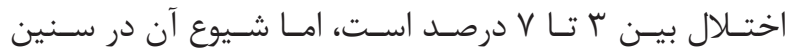

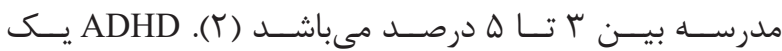

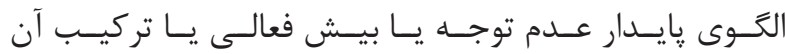

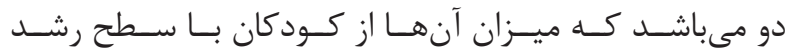

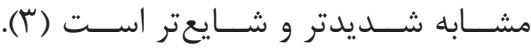

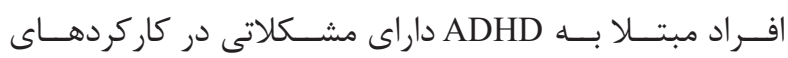

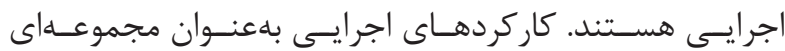

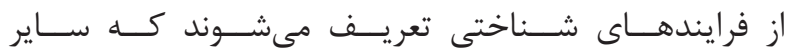

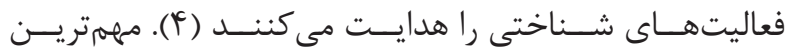

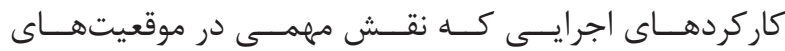

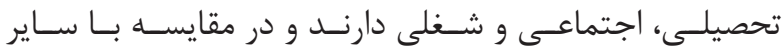

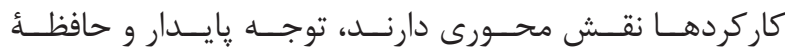

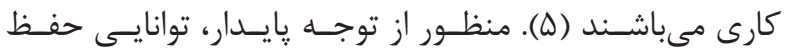

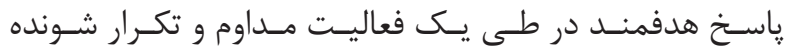

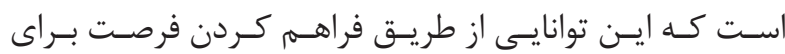

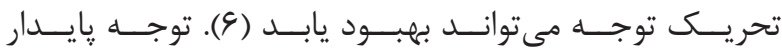

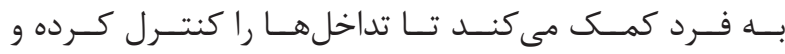

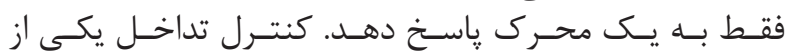

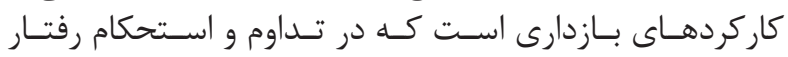

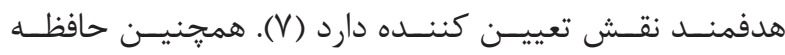

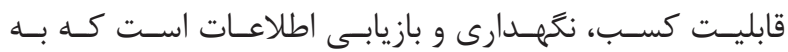

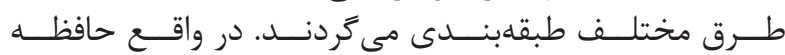

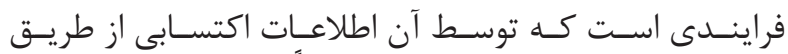

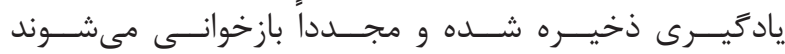

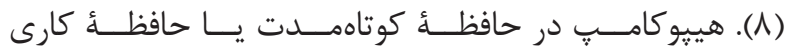

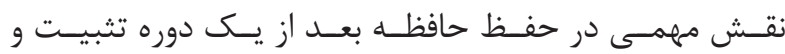

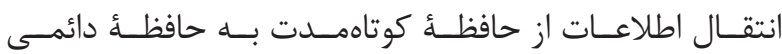

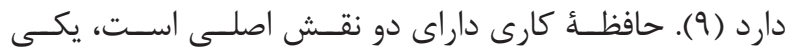

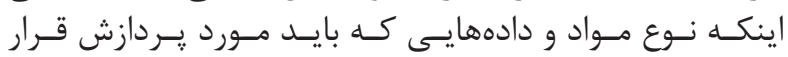

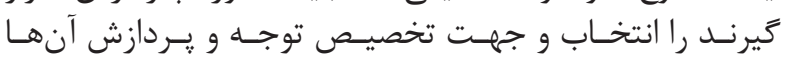

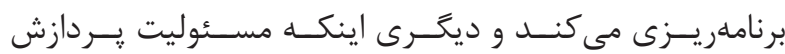

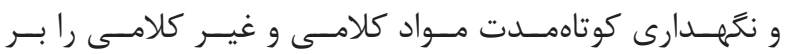

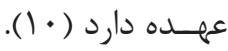

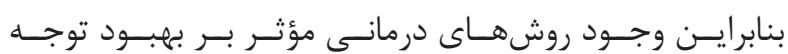

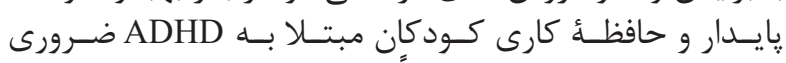

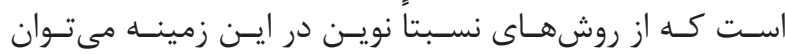

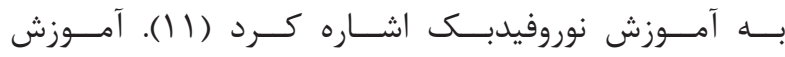

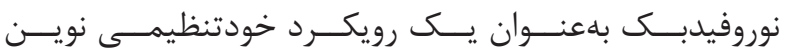

${ }^{1}$ Attention deficit/ hyperactive disorder

${ }^{2}$ Electro encephalo graphy 


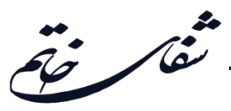

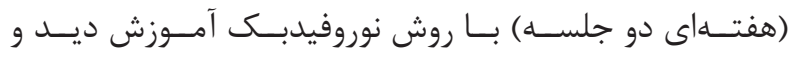

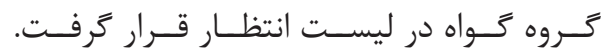

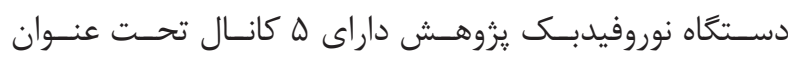

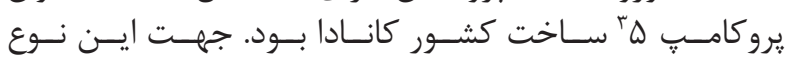

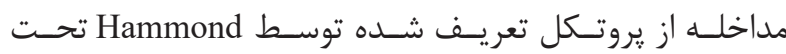

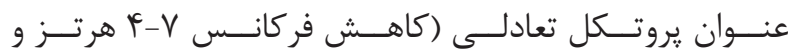

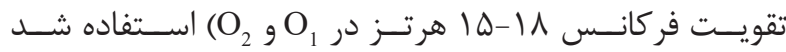

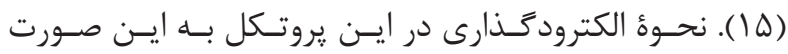

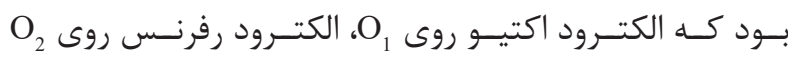

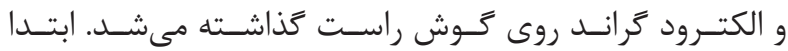

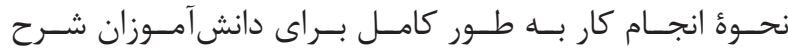

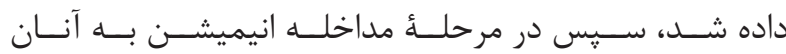

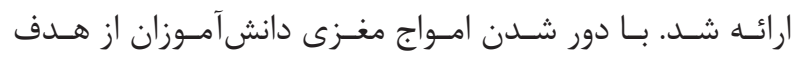

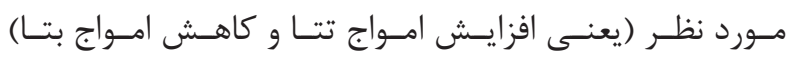

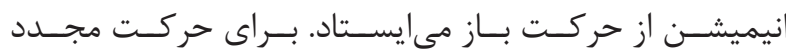

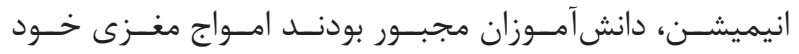

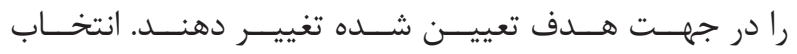

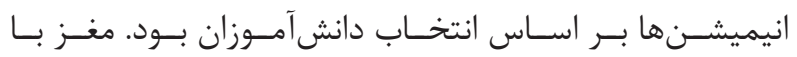

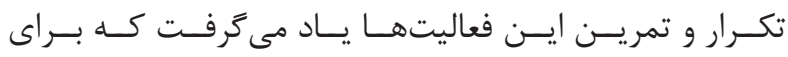

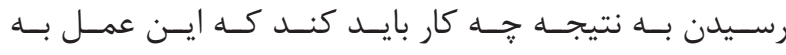

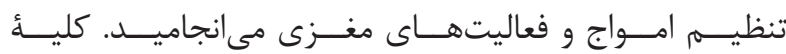

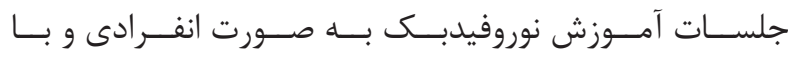

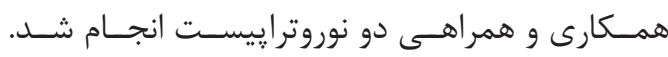

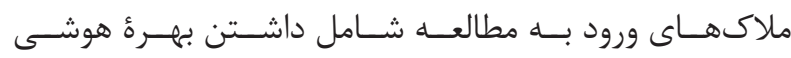

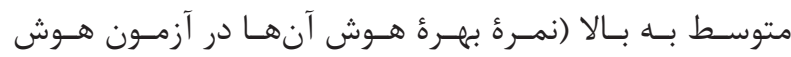

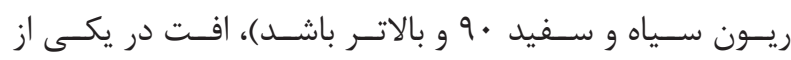

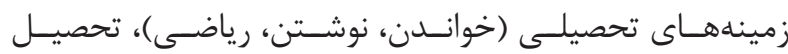

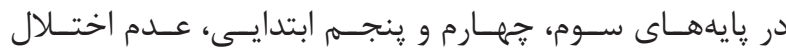

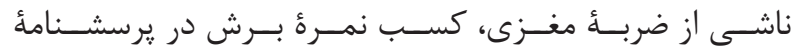

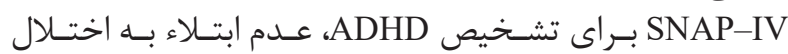

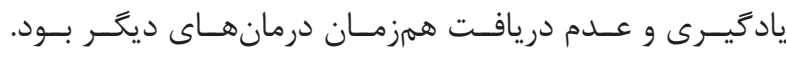

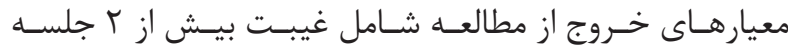

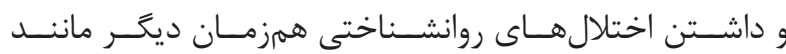

$$
\text { صـرع و غيـره بـود. }
$$

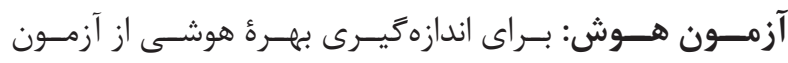

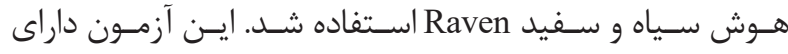

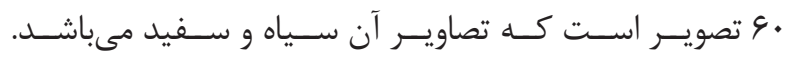

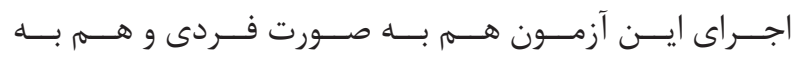

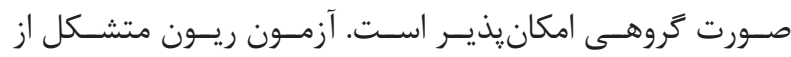

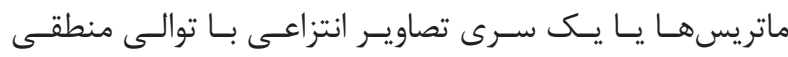

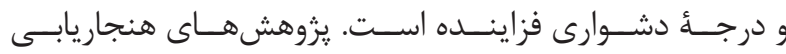

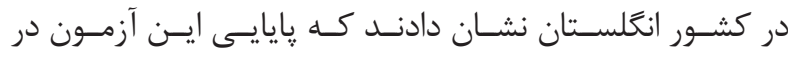

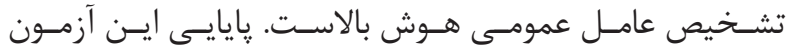

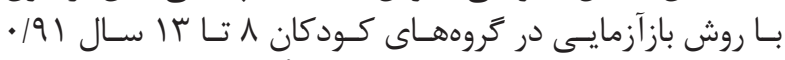

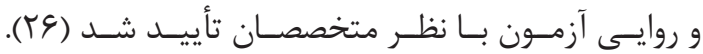

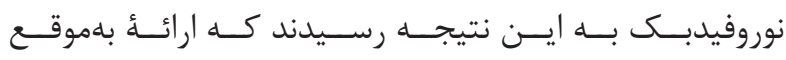

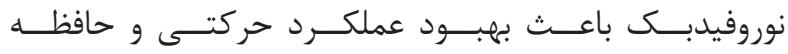

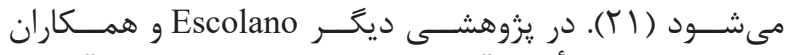

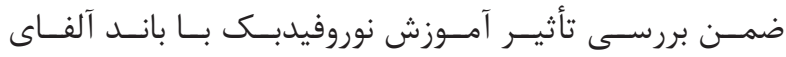

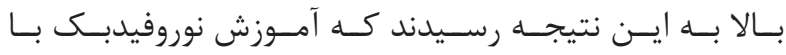

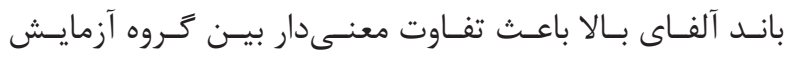

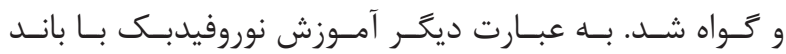

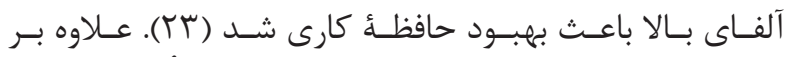

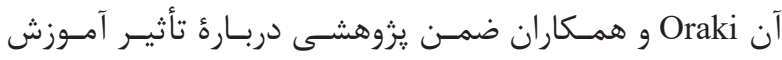

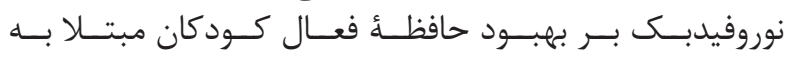

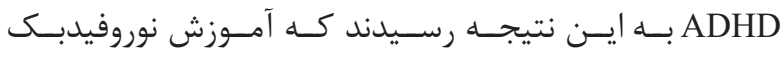

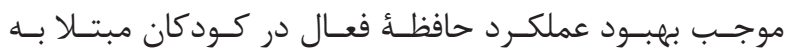

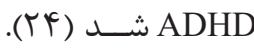

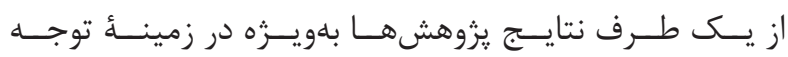

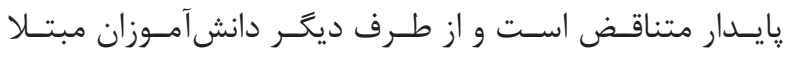

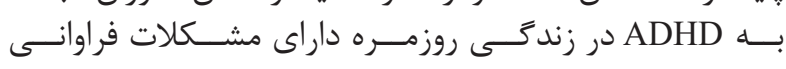

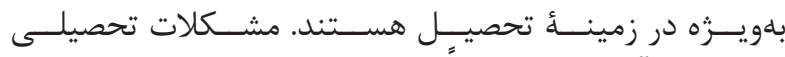

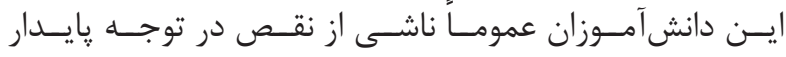

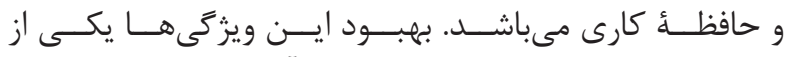

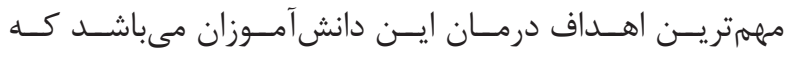

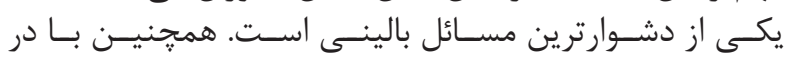

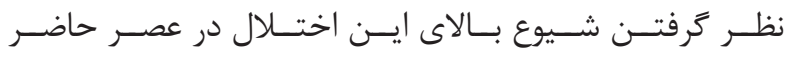

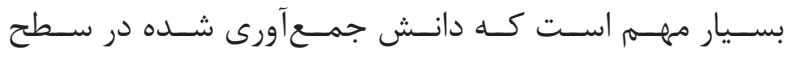

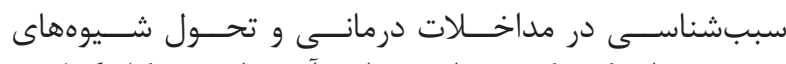

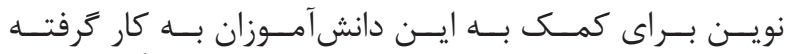

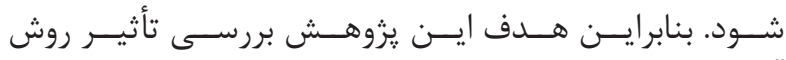

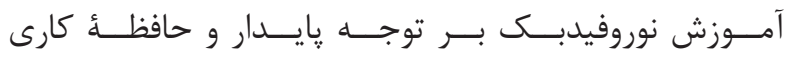

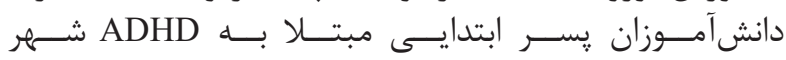

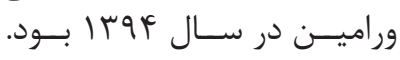

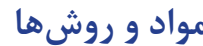

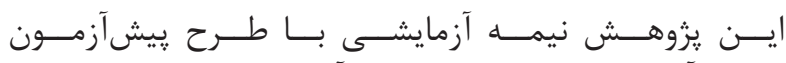

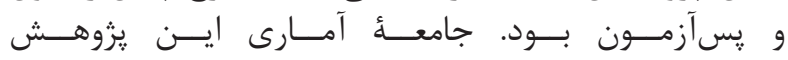

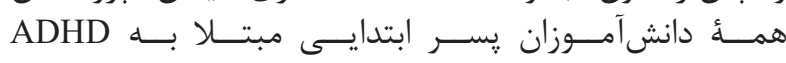

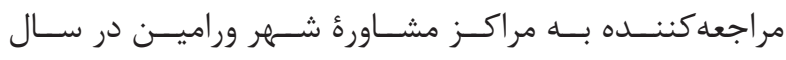

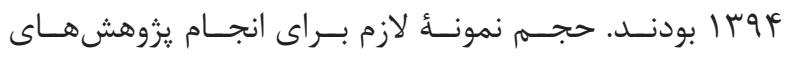

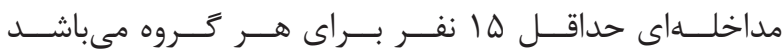
(TQ)

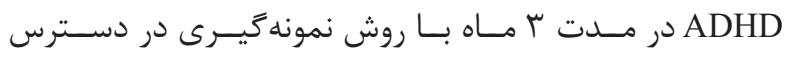

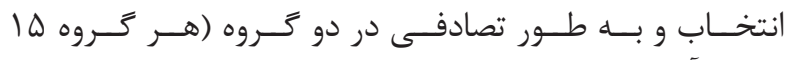

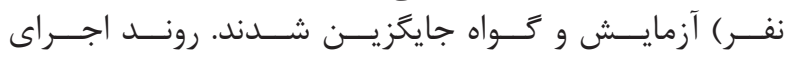

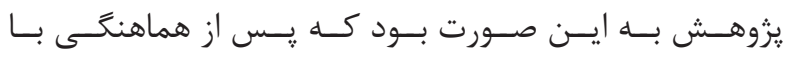

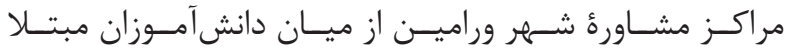

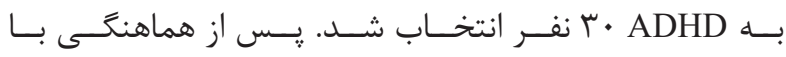

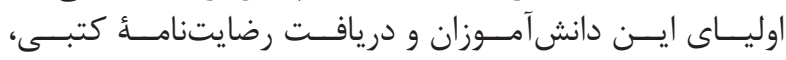

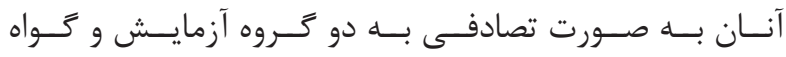

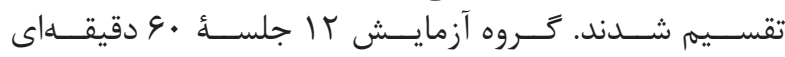




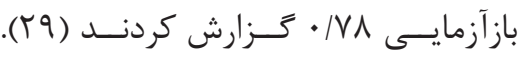

\section{تجزيه و تحليل دادهها}

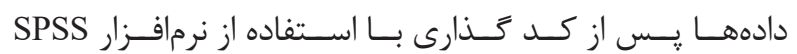

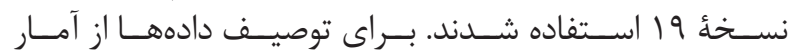

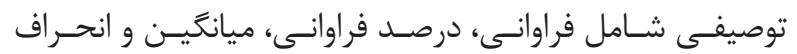

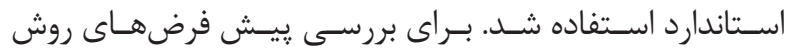

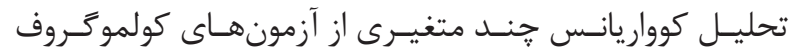

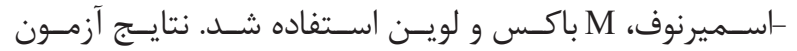

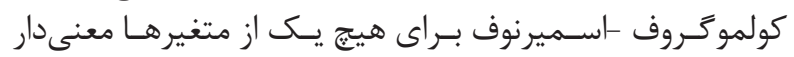

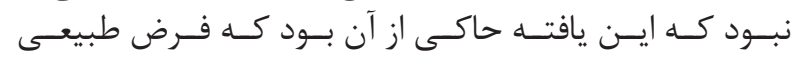

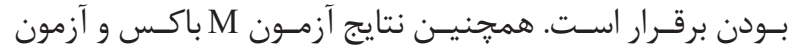

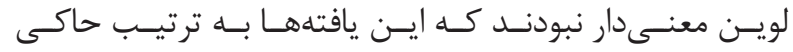

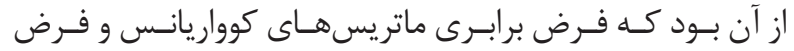

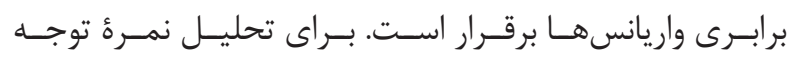

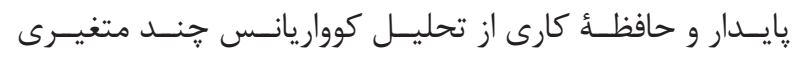

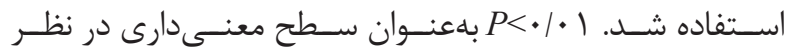

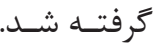

يافتهها

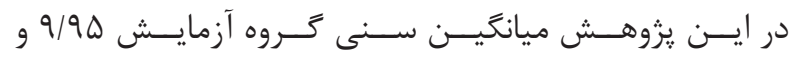

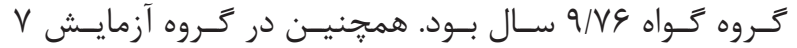

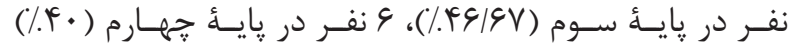

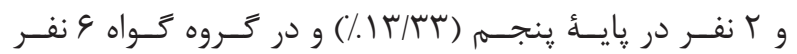

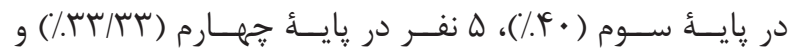

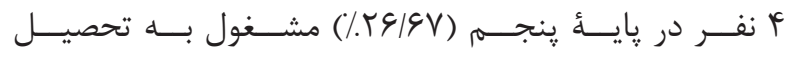

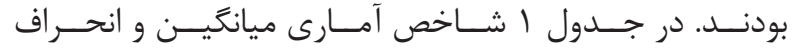

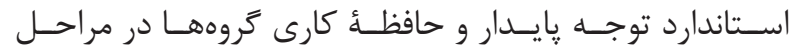

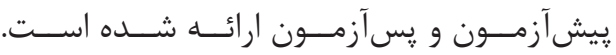

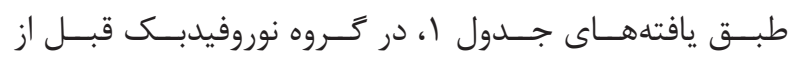

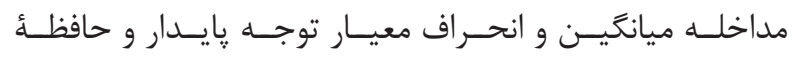

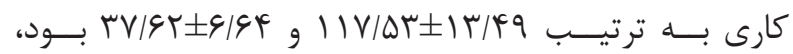

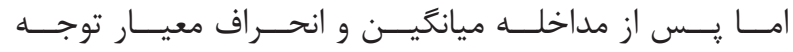

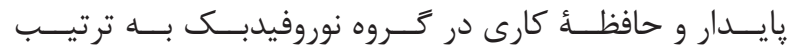

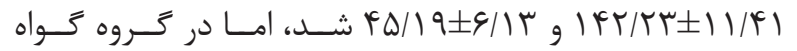

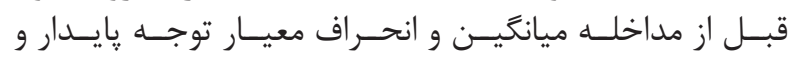

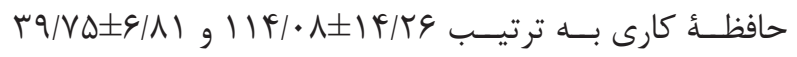

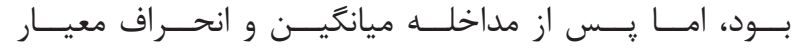

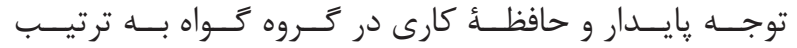

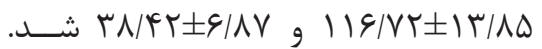

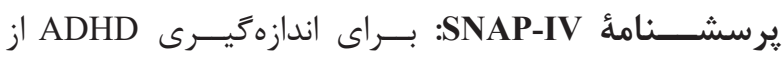

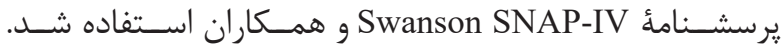

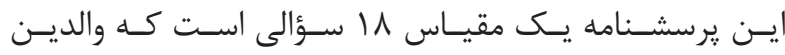

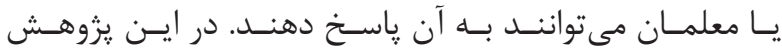

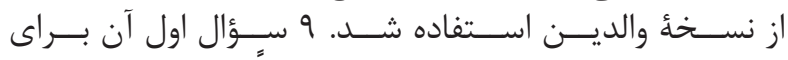

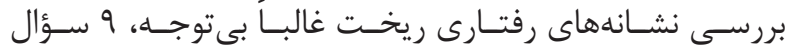

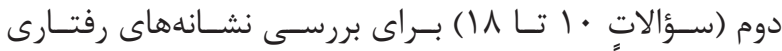

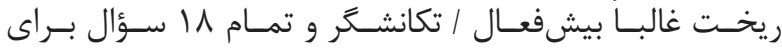

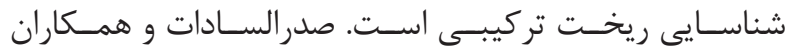

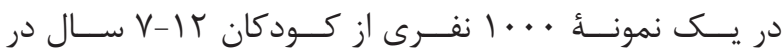

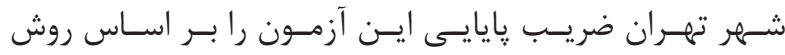

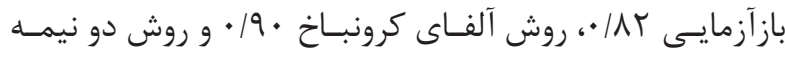

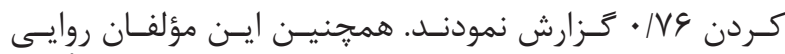

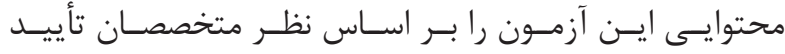

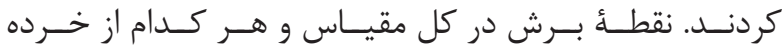

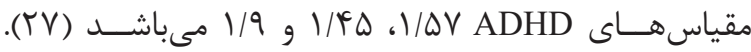

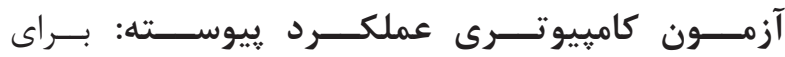

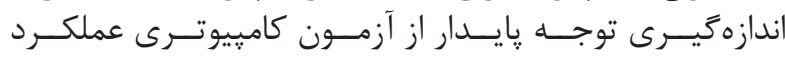

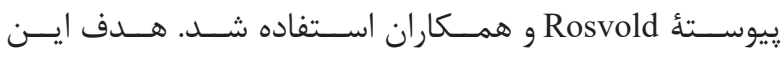

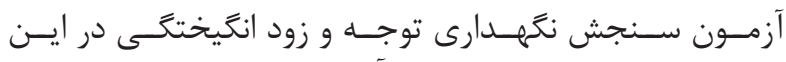

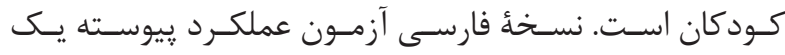

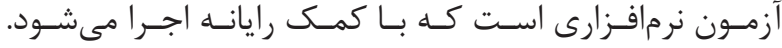

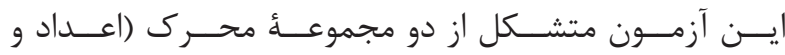

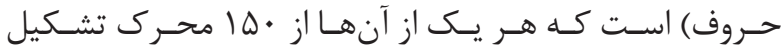

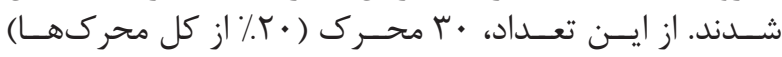

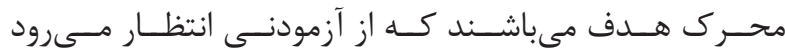

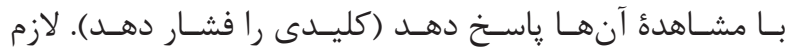

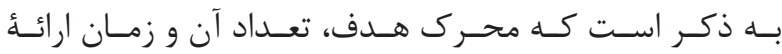

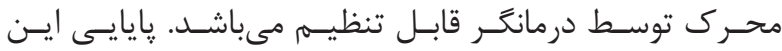

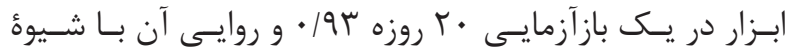

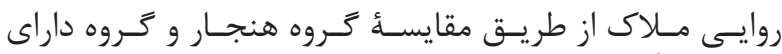

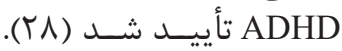

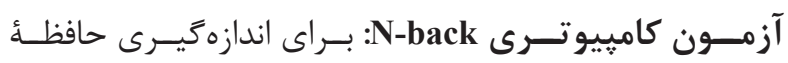

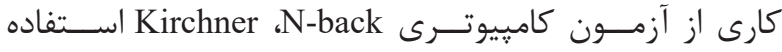

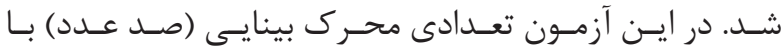

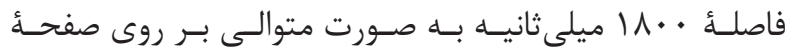

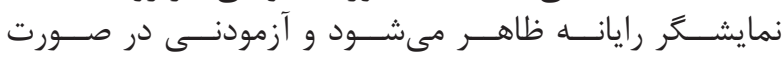

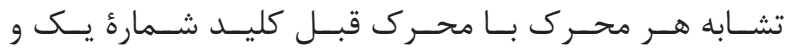

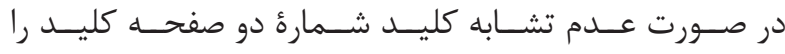

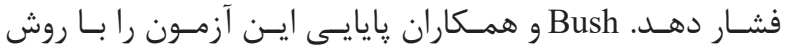

جدول ا- ميانكَين و انحراف استاندارد كَروها در مراحل يِيشآزمون و پِّآزمون.

\begin{tabular}{|c|c|c|c|c|c|c|c|c|c|}
\hline \multicolumn{4}{|c|}{ حافظة كارى } & \multicolumn{4}{|c|}{ توجه هايدار } & \multirow{3}{*}{ تعداد } & \multirow{3}{*}{ كروهها } \\
\hline \multicolumn{2}{|c|}{ يسآزمون } & \multicolumn{2}{|c|}{ بيش/آزمون } & \multicolumn{2}{|c|}{ يسآزمون } & \multicolumn{2}{|c|}{ ييش آزمون } & & \\
\hline $\mathrm{SD}$ & M & SD & M & SD & M & $\mathrm{SD}$ & M & & \\
\hline $9 / 1 \pi$ & $4 \Delta / 19$ & $9 / 94$ & TV/GT & $\mid 1 / 41$ & IFT/KT & $1 r / 49$ & $11 \mathrm{~V} / \Delta \mathrm{H}$ & 10 & نوروفيدبك \\
\hline GIAV & TNKT & $9|\wedge|$ & एq/v & $\mid r / \Lambda \Delta$ & $119 / V T$ & $\mid F / T G$ & $\| \mathrm{F} / \cdot \Lambda$ & 10 & كواه \\
\hline
\end{tabular}


بحث و نتيجه

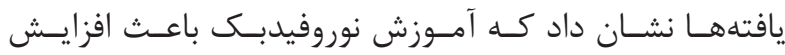

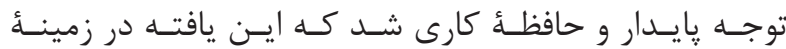

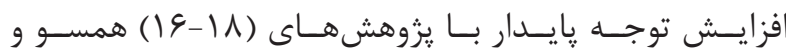

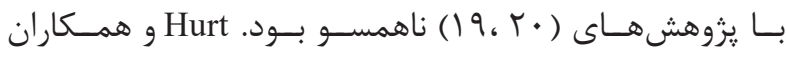

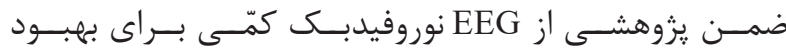

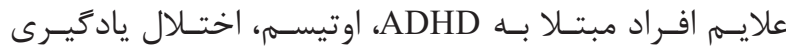

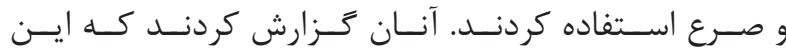

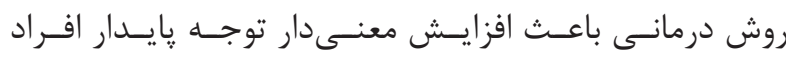

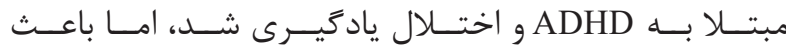

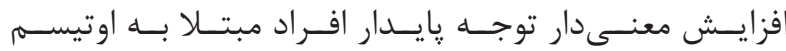

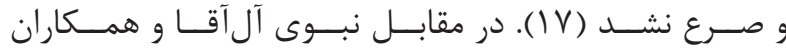

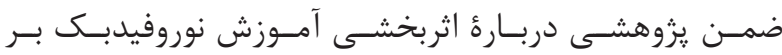

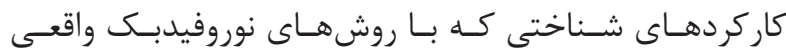

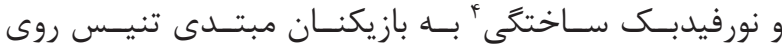

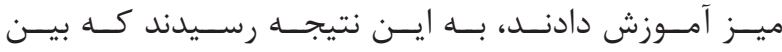

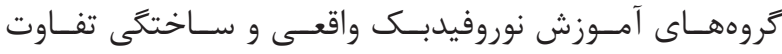

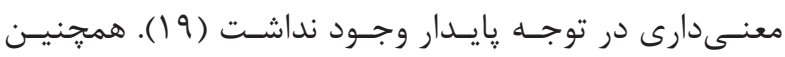

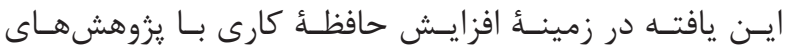

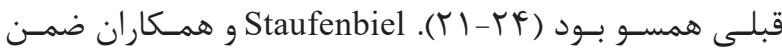

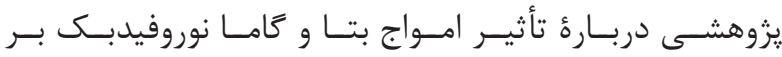

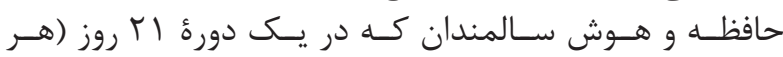

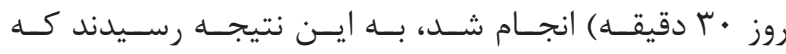

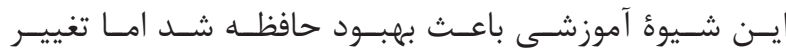

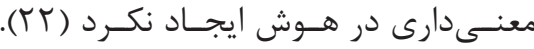

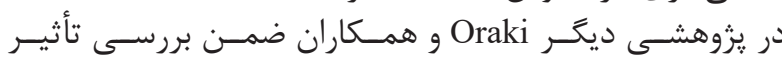

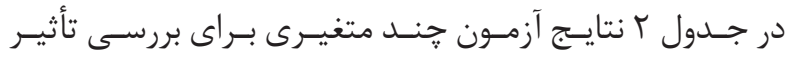

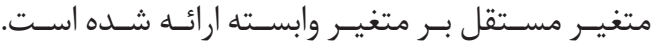

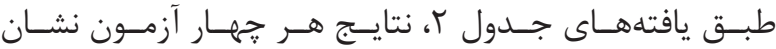

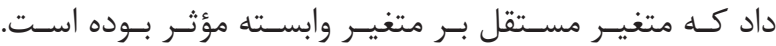

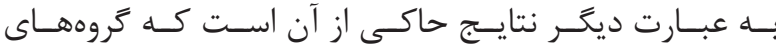

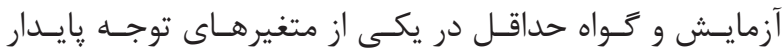

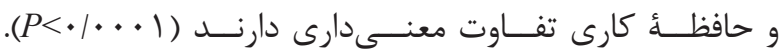

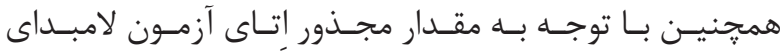
ويلكــز (FVD

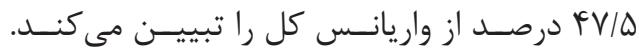

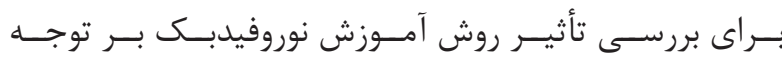

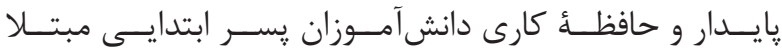

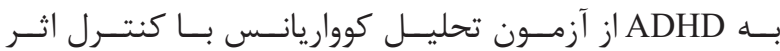

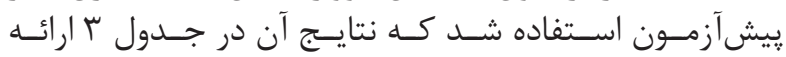

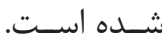

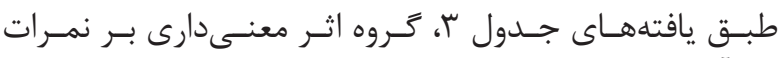

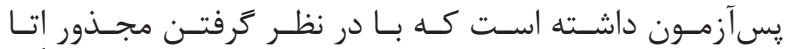

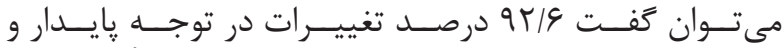

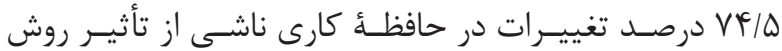

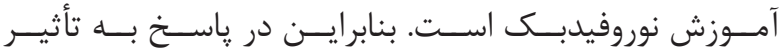

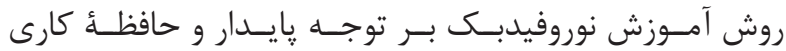

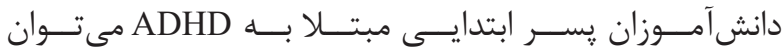

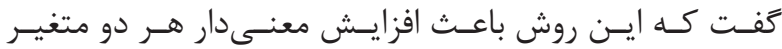

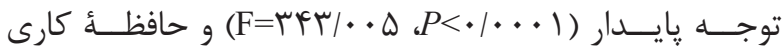

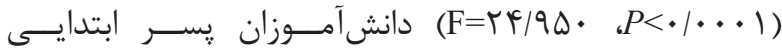
مبتـلا بـه ADHD شــده اسـت.

جدول r- نتايج آزمون קُند متغيرى براى متغيرهاى توجه پايدار و حافظئ كارى.

\begin{tabular}{|c|c|c|c|c|c|}
\hline مجذور إِا (PES) & معنى دارى & F & مقدار & آزمونها & متغير مستقل \\
\hline - Mar & $\cdot 1 \cdot \cdots 1$ & If/rrq & $\cdot|9 V|$ & اثر پييلايیى & \multirow{4}{*}{ روش درمانى } \\
\hline$\cdot / \& V A$ & $\cdot 1 \cdots \cdot 1$ & rT/. rF & ./rYq & لامبداى ويلكز & \\
\hline.$/ D F q$ & $\cdot 1 \cdots+1$ & HI/DSV & $r / 1 \wedge \Delta$ & اثر هاتلينَ & \\
\hline.$/ 2 I K$ & $.1 \cdots \cdot 1$ & 8N/19T & T/IG. & بزركترين ريشهروى & \\
\hline
\end{tabular}

جدول با- نتايج تفكيكى تحليل كوواريانس گروههاى نوروفيدبك و گواه در توجه يايدار و حافظةٌ كارى.

\begin{tabular}{|c|c|c|c|c|c|c|c|}
\hline مجذور إتا & مقلار & آمارة: F & ميانگين منورات & درجه & مجنموع مجورات & منبع اثر & متغيرهاى وابسته \\
\hline$\cdot / 4 \Delta q$ & $\cdot / \cdots 1$ & ITI/AD. & GY9V/D19 & 1 & GrgV/DI9 & هيش آزمون & \multirow{4}{*}{ توجه بايدار } \\
\hline \multirow[t]{3}{*}{.1949} & $\cdot / \cdots 1$ & TFT/. D & $\mid q T \cdot F / V q 1$ & 1 & $\mid 94 \cdot k / V 91$ & كروه & \\
\hline & & & FV/DTD & tr & ITAT/KOT & واريانس خطا & \\
\hline & & & & $r$ r. & TFATI/TG. & واريانس كل & \\
\hline (4t) & $\cdot / \cdot 1$ & $9 /$ T\& & $|V F / T| G$ & 1 & $|V Y / T| G$ & يَيش آزمون & \multirow{4}{*}{ حافظة كارى } \\
\hline \multirow[t]{3}{*}{ IVFa } & $\cdot / \cdots 1$ & $r F / q_{Q}$. & FGQ/rT^ & 1 & Fq9/rrی & كروه & \\
\hline & & & $\mid N / \Lambda \cdot V$ & tr & $\Delta \cdot V / \Lambda \cdot r$ & واريانس خطا & \\
\hline & & & & r. & |rgF/qFI & واريانس كل & \\
\hline
\end{tabular}




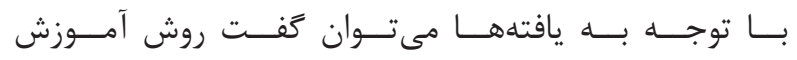

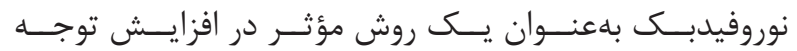

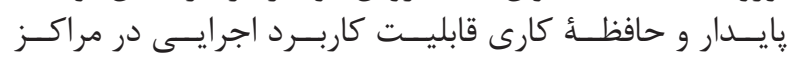

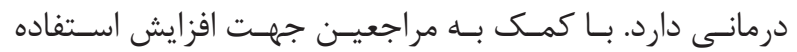

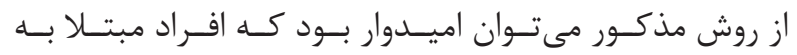

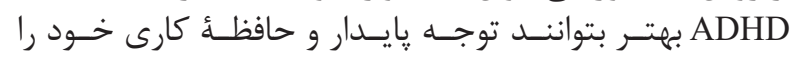

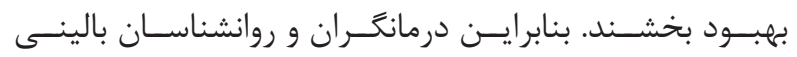

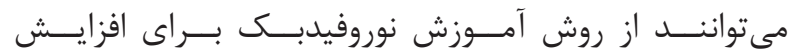

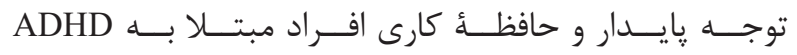

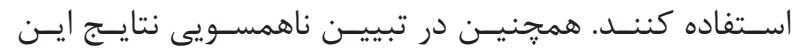

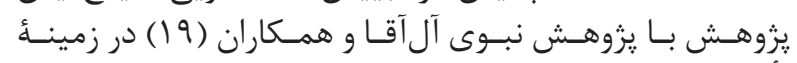

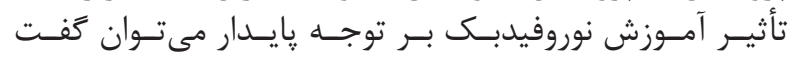

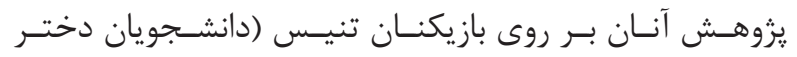

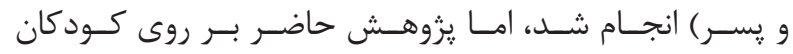

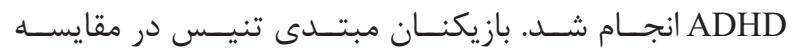

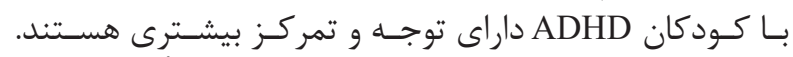

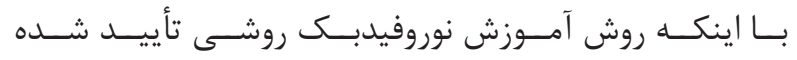

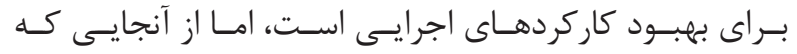

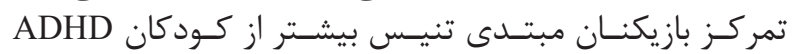

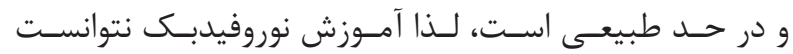

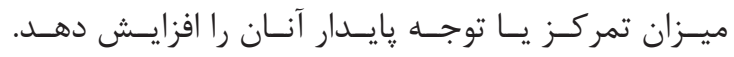

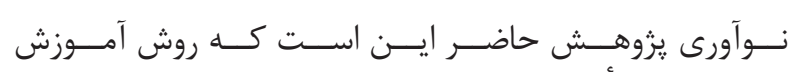

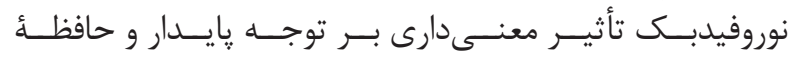

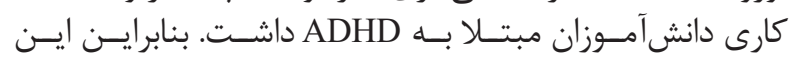

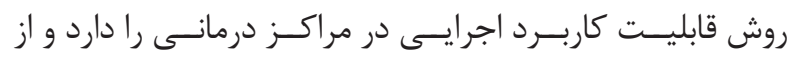

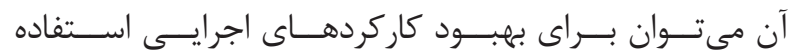

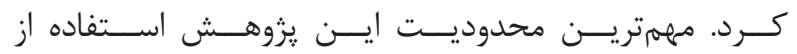

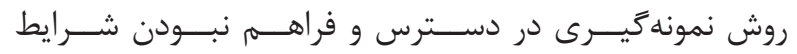

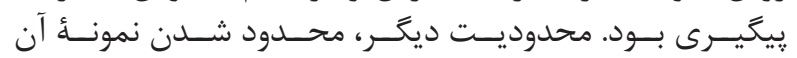

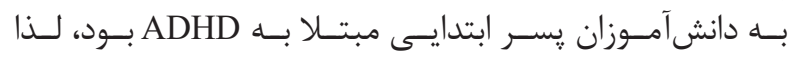

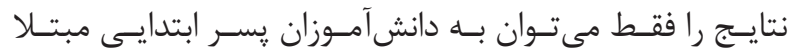

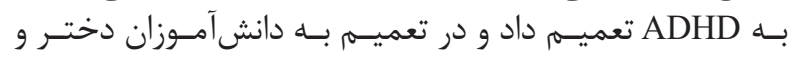

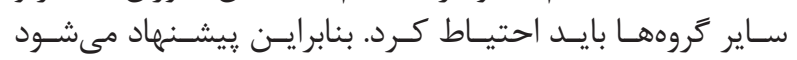

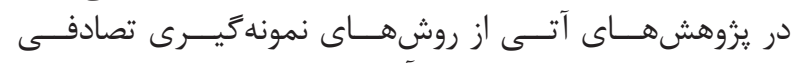

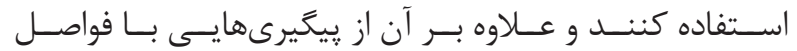

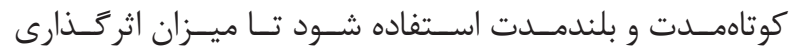

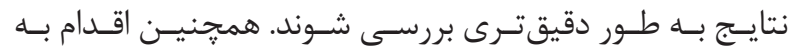

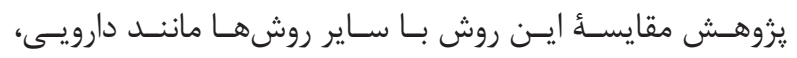

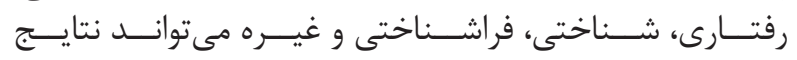

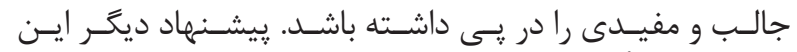

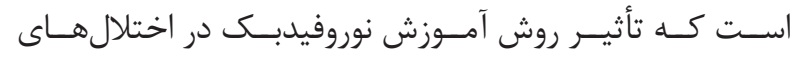

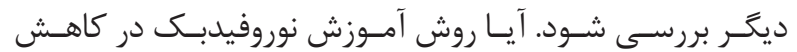

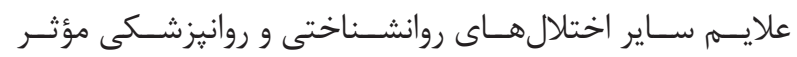

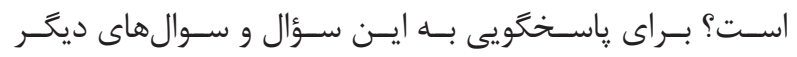

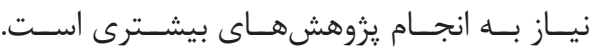

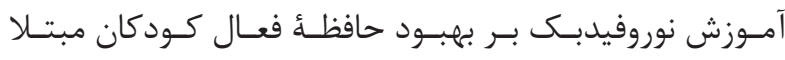

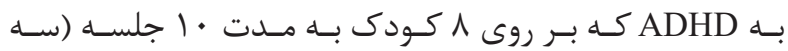

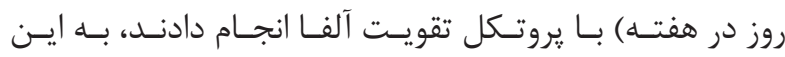

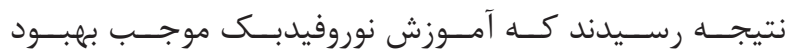

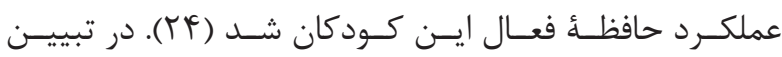

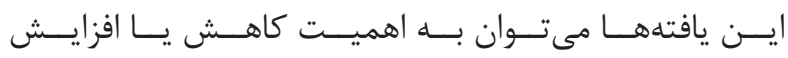

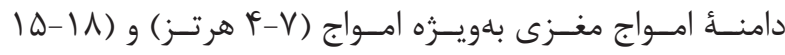

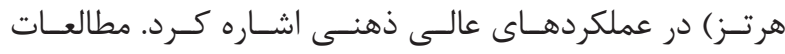

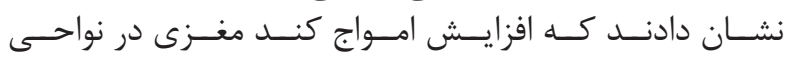

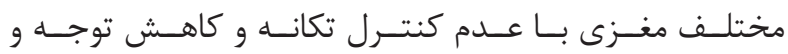

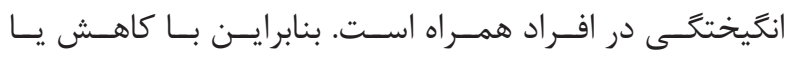

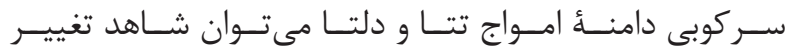

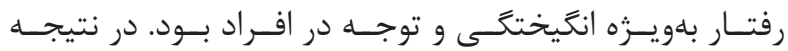

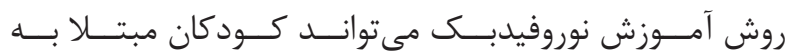

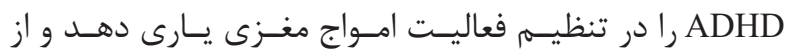

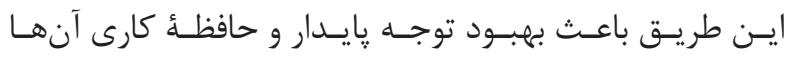

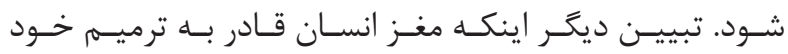

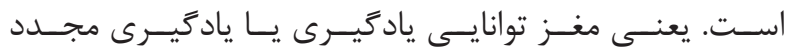

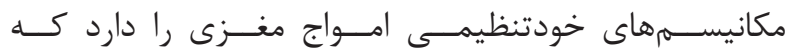

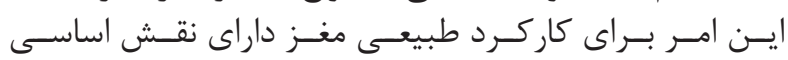

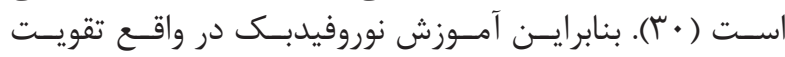

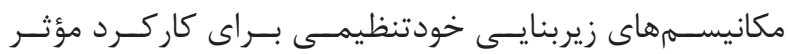

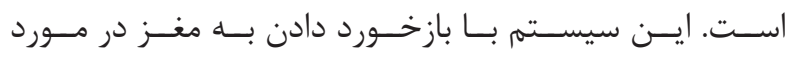

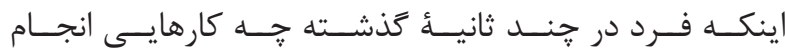

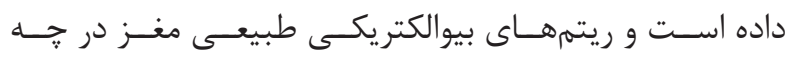

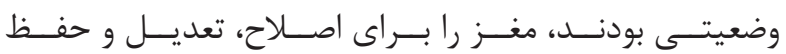

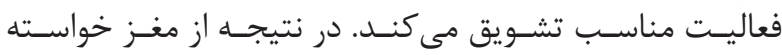

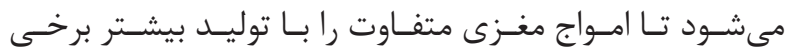

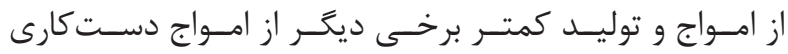

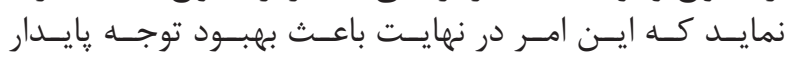

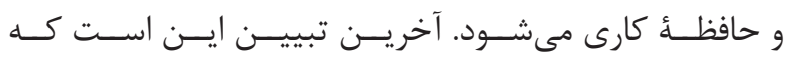

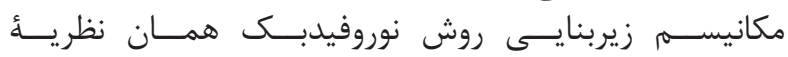

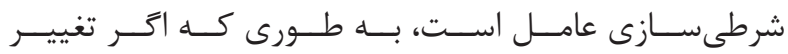

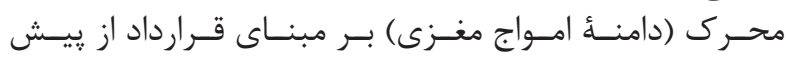

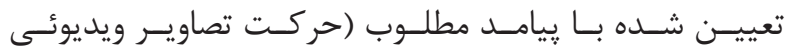

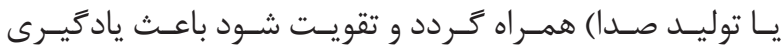

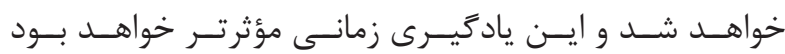

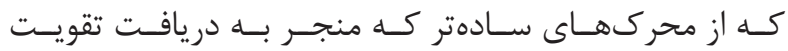

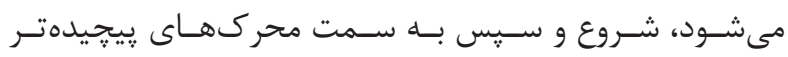

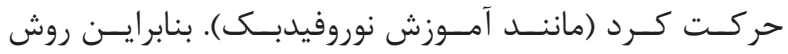

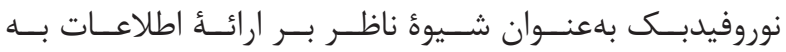

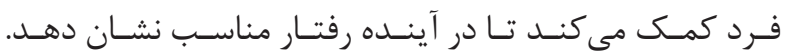

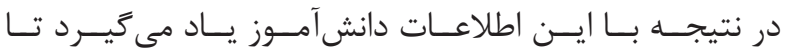

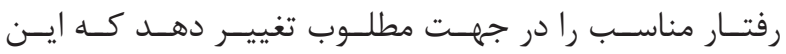

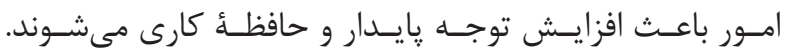


1. Fazel M, Almasi Dooghaee M, Ghasemi F, Hebrani P, Ashrafzadeh F. Evaluation of the quality of life and the frequency of attention deficit hyper activity disorder in children with epilepsy. Shefaye Khatam. 2015; 3(4): 65-72.

2. Poon K, Ho CS. Risk-taking propensity and sensitivity to punishment in adolescents with attention deficit and hyperactivity disorder symptoms and/or reading disability. Res Dev Disa. 2016; 53-54(6): 296-304.

3. Angst J, Gamma A, Rossler W, Ajdacic V, Klein DN. Long-term depression versus episodic major depression: results from the prospective Zurich study of a community sample. J Affective Disor. 2009; 115(1-2): 112-21.

4. Miller M, Hinshaw SP. Does childhood executive function predict adolescent functional outcomes in girls with ADHD? J Abnormal Child Psychol. 2010; 38(3): 315-26.

5. Najarzadegan M, Nejati V, Amiri N, Sharifian M. Effect of cognitive rehabilitation on executive function (working memory and attention) in children with attention deficit hyperactivity disorder. J Rehab Med. 2015; 4(2): 97-108.

6. Khalife N, Kantomaa M, Glover V, Tammelin T, Laitinen J, Ebeling H, et al. Childhood attention-deficit/ hyperactivity dis-order symptoms are risk factors for obesity and physical in activity in adolescence. J Am Acad Child Adolesc Psychiat. 2014; 53: 425-36.

7. Wang S, Yang Y, Xing W, Chen J, Liu C, Luo X. Altered neural circuits related to sustained attention and executive control in children with ADHD: an event-related fMRI study. Clin Neurophysio. 2013; 124(11): 2181-90.

8. Alivand F, Karimzadeh F. The effect of exercise on the memory improvement: a review of cellular and molecular mechanisms. Shefaye Khatam. 2015; 3(4): 123-30.

9. Mohammadzadeh E, Alipour F, Khallaghi B. Evaluation of spatial memory impairment after intracerebroventricular streptozocin injection in adult rats. Shefaye Khatam. 2014; 2(1): 40-5.

10. Kasper LJ, Alderson RM, Hudec KL. Moderators of working memory deficits in children with attentiondeficit/hyperactivity disorder (ADHD): a meta-analytic review. Clin Psychol Rev. 2012; 32(7): 605-17.

11. Wang JR, Hsieh S. Neurofeedback training improves attention and working memory performance. Clin Neurophysio. 2013; 124(12): 2406-20.

12. Simkin DR, Thatcher RW, Lubar J. Quantitative EEG and neurofeedback in children and adolescents: anxiety disorders, depressive disorders, comorbid addiction and attention deficit/ hyperactivity disorder, and brain injury. Child Adole Psychi Clin North Ame. 2014; 23(3): 427-64.

13. Niv S. Clinical efficacy and potential mechanisms of neurofeedback. Pers Indiv Differ. 2013; 54(6): 676-86.

14. Peeters F, Oehlen M, Ronner J, van Os J, Lousberg R. Neurofeedback as a treatment for major depressive disorder: a pilot study. Plos One. 2014; 9(3): 918-37.

15. Sterman MB, Egner T. Foundation and practice of neurofeedback for the treatment of epilepsy. Appl Psychophy Bio. 2006; 31(1): 21-35.

16. Mayer K, Blume F, Wyckoff SN, Brokmeier LL, Strehl U. Neurofeedback of slow cortical potentials as a treatment for adults with attention deficit/hyperactivity disorder. Clin Neurophysio. 2016; 127(2): 1374-86.

17. Hurt E, Arnold LE, Lofthouse N. Quantitative EEG neurofeedback for the treatment of pediatric attentiondeficit/hyperactivity disorder, autism spectrum disorders, learning disorders, and epilepsy. Child Adolescent Psychiat Clin North Am. 2014; 22(3): 465-86.

18. Arns M, Drinkenburg W, Kenemans JL. The effects QEEG-informed neurofeedback in ADHD: an openlabel pilot study. Appl Psychophy Bio. 2012; 10: 91-4.

19. Nabavi Alagha F, Naderi F, Heidari AR, Ahadi $\mathrm{H}$, Nazari MA. The effectiveness of neurofeedback training on cognitive function. Thoug Behav Clin Psychol. 2012; 7(4): 27-36.

20. Logemann HN, Lansbergen MM, VanOs TW, Bocker KB, Kenemans JL. The effectiveness of EEG- feedback on attention, impulsivity and EEG: a sham feedback controlled study. Neuro Lett. 2010; 19(1): 49-53.

21. Scharnowski F, Veit R, Zopf R, Studer P, Bock S, et al. Manipulating motor performance and memory through real-time fMRI neurofeedback. Bio Psychol. 2015; 108(5): 85-97.

22. Staufenbiel SM, Brouwer AM, Keizer AW, VanWouwe NC. Effect of beta and gamma neurofeedback on memory and intelligence in the elderly. Bio Psychol. 2014; 95(1): 74-85. 
23. Escolano C, Aguilar M, Minguez, J. EEG-based upper alpha neurofeedback training improves working memory performance. Eng Med Biol Soc. 2011, 30(3): 2327-30.

24. Oraki M, Rahmanian M, Tehrani N, Heidari Sh. Effective of neuorofeedback on improvement working memory in children with attention deficit and hyperactivity disorder. Neuropsychol. 2015; 1(1): 4151.

25. Delavar A. Theoretical and practical research in the humanities and social sciences. $7^{\text {th }}$ ed. Tehran: Roshd Publisher; 2008.

26. Sadrolsadat SJ, Hoshyary Z, zamani R, Sadrolsadat $\mathrm{L}$. The profile of the parent rating scale psychometrics. Rehabi J. 2007; 8(3): 59-65.
27. Barkley RA. The important role of executive functioning and self- regulation in ADHD. J Child Neuropsy. 2011; 113(21): 41-56.

28. Hadyanfar H, Najjarian B, Shokerkon H, Mehrabi Zadeh Honarmand M. Procurement and construction of Persian form a continuous performance test. J Psychol. 2000; 388-404.

29. Bush G, Spencer TJ, Holmes J, Shin LM, Valera EM, Seidman LJ, et al. Functional magnetic resonance imaging of methylphenidate and placebo in attentiondeficit/hyperactivity disorder during the multi-source interference task. Arch Gen Psychiat. 2008; 65(1): 102-14.

30. Demos JN. Getting started with neurofeedback. New York: Norton \& Company Publisher. 2005. 